\title{
Thermal Conductivity of 3D Boron-based Covalent Organic Frameworks from Molecular Dynamics Simulations
}

\author{
Yazhou Liu ${ }^{\dagger}$, Yanhui Feng ${ }^{*}, *$, Zhi Huang ${ }^{\dagger}+$, Xinxin Zhang ${ }^{\dagger} \neq$ \\ $\uparrow$ School of Mechanical Engineering, University of Science and Technology Beijing, \\ Beijing 100083, China \\ + Beijing Key Laboratory of Energy Saving and Emission Reduction for \\ Metallurgical Industry, University of Science and Technology Beijing, Beijing \\ 100083, China \\ Corresponding Author \\ * Yanhui Feng. E-mail: yhfeng@me.ustb.edu.cn. Tel: 86-010-62334971
}




\section{Validation of the simulation method}

To prove the validity of the simulation method, we calculated the thermal conductivity of ZIF-8 to use the force field developed by Zheng et al.. ${ }^{1}$ Fig. $1 \mathrm{~S}$ shows the crystal structure of ZIF-8.

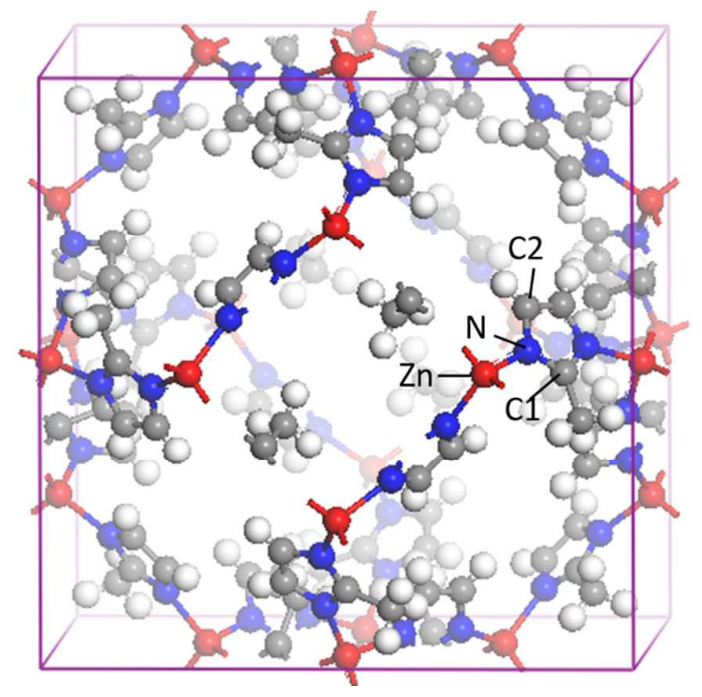

Fig.1S Crystal structure of ZIF-8. Color coding: Zn, red; N, blue; C, gray; H, white.

Normalized heat current autocorrelation function and thermal conductivity at $300 \mathrm{~K}$ as a function of correlation time are given in Fig.2S (a) and (b). Normalized HCACF decays to approximately zero at a time much shorter than the correlation time of eight ps, so the correlation time of eight ps is long enough for the calculation of thermal conductivity. The thermal conductivity converges more slowly and reaches a constant value at approximately eight ps. The estimated thermal conductivity of ZIF-8 is 0.170 $\mathrm{W} /(\mathrm{m} \cdot \mathrm{K})$ at $300 \mathrm{~K}$, which is a little higher than that of Zhang's simulation $(0.165 \mathrm{~W} /$ $(\mathrm{m} \cdot \mathrm{K}))$. This is attributed to the use of different force field parameters. 

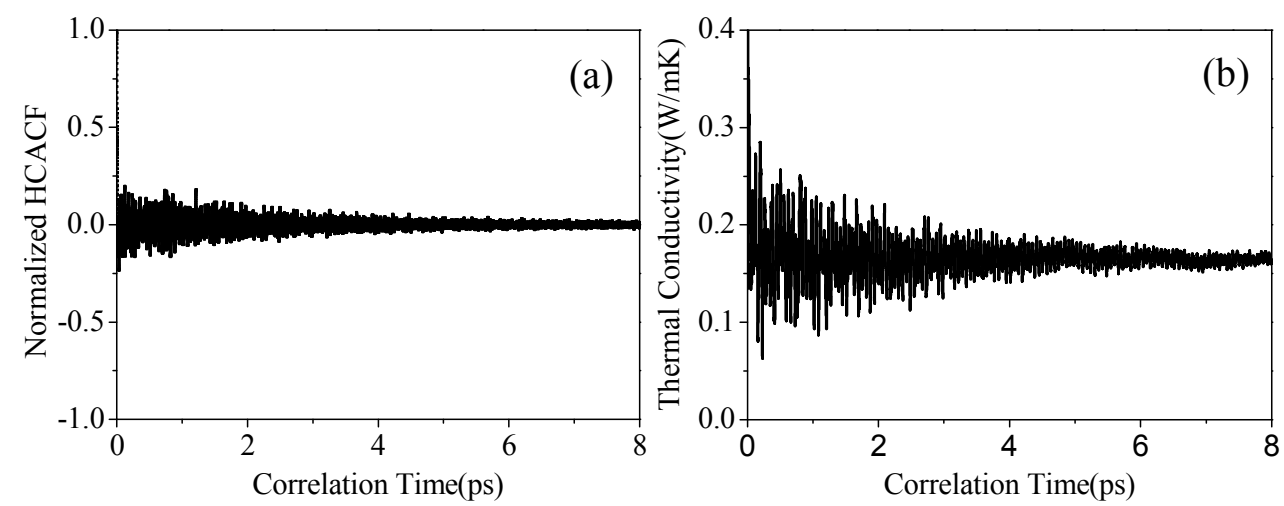

Fig.2S (a) Normalized heat current autocorrelation function and (b) thermal conductivity at $300 \mathrm{~K}$ as a function of correlation time.

Fig. 3S shows temperature effect on thermal conductivity of ZIF-8 of this study and Zhang's simulation. ${ }^{2}$ Compared with Zhang's simulation result, our simulation values are a little higher. However, different force field results in the slight difference. So our simulation method is reliable.

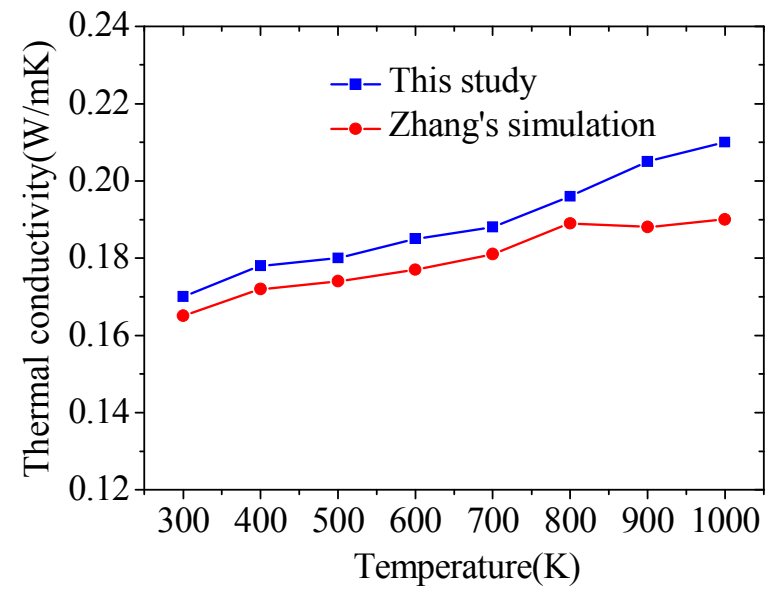

Fig. 3S Temperature effect on thermal conductivity of ZIF-8.

We used overlap energy to quantitatively analyze the relationship between the crystal structure and the low thermal conductivity. To guarantee the feasibility of this method, we calculated the overlap energy of two bonded atoms in ZIF-8. Fig.4S shows the overlap energy of per two bonded atoms in ZIF-8. Overlap energy between $\mathrm{Zn}$ and $\mathrm{N}$ atoms is the minimum. Zhang ${ }^{2}$ obtained that the temperature effect is attributed to the enhanced overlap in the vibrational density of states between $\mathrm{Zn}$ and 
$\mathrm{N}$ atoms. It means thermal conductivity of ZIF-8 is governed by Zn-N bonds. Our results turn out that $\mathrm{Zn}-\mathrm{N}$ bonds act as a bottleneck in ZIF-8, in accord with the hypothetical result of Zhang's.

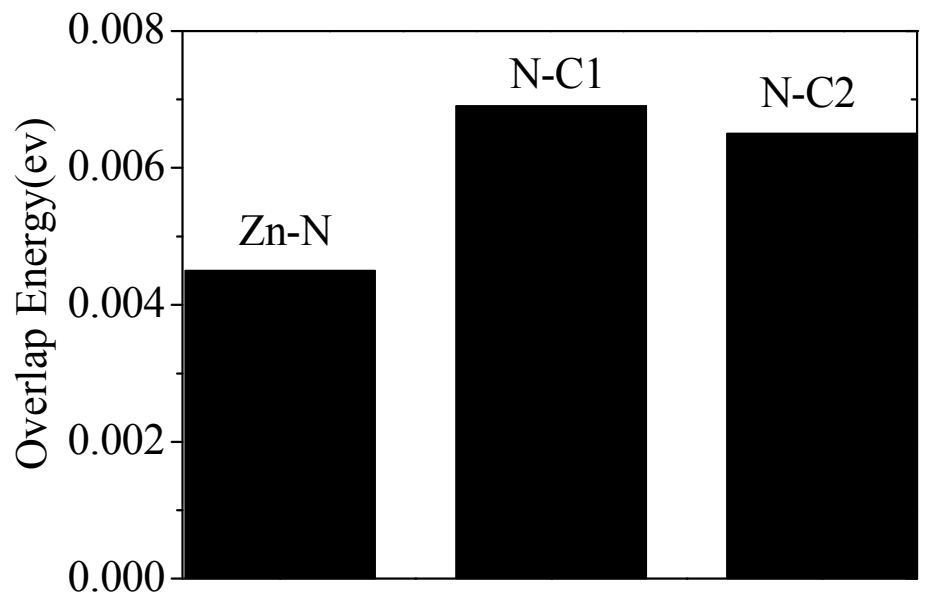

Fig.4S Overlap energy of per two bonded atoms in ZIF-8.

\section{Force field expression}

In 2012, Rochus Schmid and coworkers have developed a force field for boron based covalent organic frameworks by a first-principles derived force field. ${ }^{3}$ In this force field, the full energy expression is given as follow:

$$
\begin{aligned}
& E_{\text {total }}=\sum_{b} E_{b}^{\text {stretch }}+\sum_{a}\left(E_{a}^{\text {bend }}+E_{a}^{\text {str-str }}+E_{a}^{\text {str-bend }}\right)+\sum_{o} E_{o}^{o p b}+\sum_{t} E_{t}^{\text {tors }}+ \\
& \sum_{n}\left(E_{n}^{\text {vdw }}+E_{n}^{\text {coul }}\right)
\end{aligned}
$$

where $\mathrm{b}$ runs over all bonds, $a$ over all bond angles, o over all out-of-plane bends on trigonal centers and t over all torsions.

$$
\begin{gathered}
E_{b}^{\text {stretch }}=\frac{1}{2} k_{b}\left(r_{b}-r_{b}^{r e f}\right)^{2}\left[1-2.55\left(r_{b}-r_{b}^{r e f}\right)+\frac{7}{12}\left(2.55\left(r_{b}-r_{b}^{r e f}\right)\right)^{2}\right] \\
E_{a}^{\text {bend }}=\frac{1}{2} k_{a}\left(\theta_{a}-\theta_{a}^{r e f}\right)^{2}\left[1-0.14\left(\theta_{a}-\theta_{a}^{r e f}\right)+5.6 \times 10^{-5}\left(\theta_{a}-\theta_{a}^{r e f}\right)^{2}-7 \times\right. \\
\left.10^{-7}\left(\theta_{a}-\theta_{a}^{r e f}\right)^{3}+2.2 \times 10^{-8}\left(\theta_{a}-\theta_{a}^{r e f}\right)^{4}\right] \\
E_{a}^{\text {str-str }}=k_{s s}\left(r_{a 1}-r_{a 1}^{r e f}\right)\left(r_{a 2}-r_{a 2}^{r e f}\right)
\end{gathered}
$$




$$
\begin{gathered}
E_{a}^{\text {str-bend }}=\left[k_{s b 1}\left(r_{a 1}-r_{a 1}^{r e f}\right)+k_{s b 2}\left(r_{a 2}-r_{a 2}^{r e f}\right)\right]\left(\theta_{a}-\theta_{a}^{r e f}\right) \\
E_{o}^{\text {opt }}=\frac{1}{2} k_{o}\left(\theta_{o}-\theta_{o}^{r e f}\right)^{2}\left[1-0.14\left(\theta_{o}-\theta_{o}^{r e f}\right)+5.6 \times 10^{-5}\left(\theta_{o}-\theta_{o}^{r e f}\right)^{2}-7 \times 10^{-7}\left(\theta_{o}-\right.\right. \\
\left.\left.\theta_{o}^{r e f}\right)^{3}+2.2 \times 10^{-8}\left(\theta_{o}-\theta_{o}^{r e f}\right)^{4}\right] \\
E_{t}^{\text {tors }}=\sum_{n} \frac{V_{t}^{n}\left[1+\cos \left(n T_{t}+T_{t}^{n}\right)\right]}{2} \\
E_{n}^{\text {coul }}=\frac{1}{4 \pi \epsilon} q_{1} q_{2} \frac{1}{d_{n}} \\
E_{n}^{v d w}=\epsilon_{n}\left[1.84 \times 10^{5} e^{\left.-12 \frac{d_{n}}{d_{n}^{0}}-2.25\left(\frac{d_{n}^{0}}{d_{n}}\right)^{6}\right]}\right.
\end{gathered}
$$

where $r_{b}$ is the bond distance, $\theta_{a}$ is the bond angle, $\theta_{o}$ is the out-of-plane angle; $k_{b}, k_{a}$ and $k_{o}$ are the force constant; $r_{b}^{r e f}, r_{a 1}^{r e f}$ and $r_{a 2}^{r e f}$ (For three connected atoms A-B-C of a bond angle $a$, the distance $r_{a 1}$ refers to the distance A-B, and $r_{a 2}$ to the distance B-C.)are the corresponding reference distance; $\theta_{a}^{r e f}$ and $\theta_{a}^{\text {ref }}$ are the reference angle; $V_{t}^{n}$ and $T_{t}^{n}$ are the energy barrier and the phase shift, respectively, for the $\mathrm{n}$-fold term with $T_{t}$ being the torsion angle of torsion $\mathrm{t} ; d_{n}$ is the distance of the two atoms of interaction $\mathrm{n}, q_{1}$ and $q_{2}$ are the atoms charges and $\epsilon_{n}$ and $d_{n}^{0}$ the well depth and minimum distance, respectively.

LAMMPS does not have a complete force field toolkit for the complete force field in the reference [3]. We wrote the codes of the force field in reference [3] by ourselves. We first find the similar force field expression before we begin to write the code. Then we amended the force field files with the .cpp and .h suffix.

\section{Force field parameters}

\section{1 non-bonding orce field parameters}

Table 1S: Point charges

\begin{tabular}{cc}
\hline \multicolumn{2}{c}{ "general charge" } \\
\hline atom & point-charge \\
\hline
\end{tabular}




\begin{tabular}{|c|c|}
\hline $\mathrm{C}\left(\mathrm{sp}^{2}\right)$ & -0.12 \\
\hline $\mathrm{H}$ & 0.12 \\
\hline \multicolumn{2}{|c|}{ A } \\
\hline atom & point-charge \\
\hline $\mathrm{O}$ & -0.627 \\
\hline $\mathrm{B}$ & 0.741 \\
\hline $\mathrm{C}[-\mathrm{B}]$ & -0.114 \\
\hline \multicolumn{2}{|c|}{ B1 } \\
\hline atom & point-charge \\
\hline $\mathrm{C}_{\text {center }}$ & 0.000 \\
\hline \multicolumn{2}{|c|}{ B2 } \\
\hline atom & point-charge \\
\hline $\mathrm{O}$ & -0.358 \\
\hline $\mathrm{B}$ & 0.449 \\
\hline $\mathrm{C}[-\mathrm{O}]$ & 0.195 \\
\hline $\mathrm{C}[-\mathrm{B}]$ & -0.123 \\
\hline \multicolumn{2}{|c|}{$\mathrm{D}[\mathrm{C}]$} \\
\hline atom & point-charge \\
\hline $\mathrm{C}\left(\mathrm{sp}^{3}\right)$ & 0.000 \\
\hline $\mathrm{C}\left(\mathrm{sp}^{2}\right)\left[-\mathrm{C}\left(\mathrm{sp}^{3}\right)\right]$ & 0.000 \\
\hline \multicolumn{2}{|c|}{$\mathrm{D}[\mathrm{Si}]$} \\
\hline atom & point-charge \\
\hline $\mathrm{Si}$ & -0.458 \\
\hline $\mathrm{C}\left(\mathrm{sp}^{2}\right)[-\mathrm{Si}]$ & 0.1145 \\
\hline
\end{tabular}

Table 2S: Van der Waals parameters

\begin{tabular}{cccc}
\hline & radius, $\AA$ & $\begin{array}{c}\text { Well depth, } \\
{[\mathrm{kcal} / \mathrm{mol}]}\end{array}$ & $\begin{array}{c}\text { Bond length } \\
\text { reduction factor }\end{array}$ \\
\hline $\mathrm{C}\left(\mathrm{sp}^{3}\right)$ & 2.040 & 0.027 & \\
\hline $\left.\mathrm{C}^{3} \mathrm{sp}^{2}\right)$ & 1.960 & 0.056 & \\
\hline $\mathrm{C}_{\text {center }}$ & 1.960 & 0.056 & \\
\hline $\mathrm{B}$ & 2.150 & 0.014 & \\
\hline $\mathrm{Si}$ & 2.290 & 0.014 & 0.923 \\
\hline $\mathrm{O}$ & 1.820 & 0.059 & \\
\hline $\mathrm{H}$ & 1.620 & 0.02 & \\
\hline
\end{tabular}

\subsection{Bonding force field parameters}




\section{Fragment A}

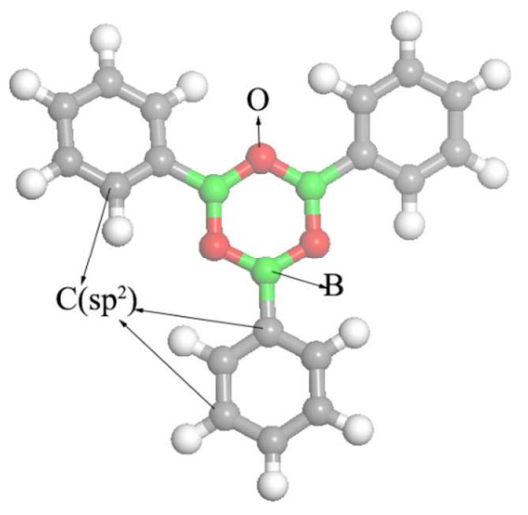

Fig.5S Fragment A (force field nomenclature)

Table 3S: Force field parameters for the fragment A. Only parameters deviating from the original MM3 parameter set are given.

\begin{tabular}{|c|c|c|}
\hline \multicolumn{3}{|c|}{ Bond stretch parameter } \\
\hline bond type & reference distance, $[\AA]$ & force constant, $[\mathrm{mdyn} / \AA]$ \\
\hline $\mathrm{O}-\mathrm{B}$ & 1.400 & 4.940 \\
\hline $\mathrm{C}\left(\mathrm{sp}^{2}\right)-\mathrm{B}$ & 1.539 & 4.670 \\
\hline \multicolumn{3}{|c|}{ In-plane angle bending parameter } \\
\hline Angle type & Reference angle, $[\mathrm{deg}]$ & $\begin{array}{l}\text { force constant, } \\
{\left[\mathrm{mdyn} \AA / \mathrm{rad}^{2}\right]}\end{array}$ \\
\hline $\mathrm{C}\left(\mathrm{sp}^{2}\right)-\mathrm{C}\left(\mathrm{sp}^{2}\right)-\mathrm{B}$ & 124.500 & 0.380 \\
\hline B-O-B & 123.500 & 0.770 \\
\hline $\mathrm{C}\left(\mathrm{sp}^{2}\right)-\mathrm{B}-\mathrm{O}$ & 118.600 & 0.660 \\
\hline O-B-O & 120.000 & 0.950 \\
\hline \multicolumn{3}{|c|}{ Out-of-plane angle bending parameter } \\
\hline oop type: central-apex & reference angle, $[\mathrm{deg}]$ & $\begin{array}{l}\text { force constant, } \\
{\left[\mathrm{mdyn} \AA / \mathrm{rad}^{2}\right]}\end{array}$ \\
\hline $\mathrm{C}\left(\mathrm{sp}^{2}\right)-\mathrm{B}$ & 0.000 & 0.060 \\
\hline $\mathrm{B}-\mathrm{C}\left(\mathrm{sp}^{2}\right)$ & 0.000 & 1.880 \\
\hline $\mathrm{B}-\mathrm{O}$ & 0.000 & 0.850 \\
\hline \multicolumn{3}{|c|}{ Torsional parameter } \\
\hline torsional type & $\mathrm{n}$ & Barrier height, $[\mathrm{kcal} / \mathrm{mol}]$ \\
\hline$C\left(\mathrm{sp}^{2}\right)-\mathrm{C}\left(\mathrm{sp}^{2}\right)-\mathrm{C}\left(\mathrm{sp}^{2}\right)-\mathrm{B}$ & 2 & 6.040 \\
\hline $\mathrm{H}-\mathrm{C}\left(\mathrm{sp}^{2}\right)-\mathrm{C}\left(\mathrm{sp}^{2}\right)-\mathrm{B}$ & 2 & 5.390 \\
\hline $\mathrm{C}\left(\mathrm{sp}^{2}\right)-\mathrm{B}-\mathrm{O}-\mathrm{B}$ & 2 & 1.440 \\
\hline B-O-B-O & 2 & 3.940 \\
\hline
\end{tabular}



$\mathrm{C}\left(\mathrm{sp}^{2}\right)-\mathrm{C}\left(\mathrm{sp}^{2}\right)-\mathrm{B}-\mathrm{O}$
2
1.760

\section{Fragment B}

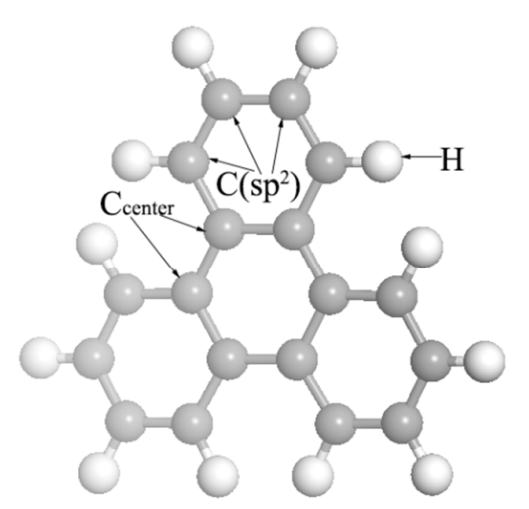

Fig.6S Fragment B (force field nomenclature)

Table 4S: Force field parameters for the fragment B.

\begin{tabular}{ccc}
\hline & Bond stretch parameter \\
\hline bond type & reference distance, $[\AA]$ & force constant, $[\mathrm{mdyn} / \AA]$ \\
\hline $\mathrm{C}\left(\mathrm{sp}^{2}\right)-\mathrm{C}_{\text {center }}$ & 1.428 & 6.686 \\
\hline $\mathrm{C}_{\text {center }}-\mathrm{C}_{\text {center }}$ & 1.449 & 6.123 \\
\hline \multicolumn{3}{c}{ In-plane angle bending parameter } \\
\hline Angle type & Reference angle, $[\mathrm{deg}]$ & force constant, \\
& & {$\left[\mathrm{mdyn} \AA / \mathrm{rad}^{2}\right]$} \\
\hline $\mathrm{C}_{\text {center }}-\mathrm{C}_{\text {center }}-\mathrm{C}_{\text {center }}$ & 126.816 & 0.505 \\
\hline $\mathrm{C}_{\text {center }}-\mathrm{C}_{\text {center }}-\mathrm{C}_{\left(\mathrm{sp}^{2}\right)}$ & 126.816 & 0.940 \\
\hline
\end{tabular}

Out-of-plane angle bending parameter

oop type: central-apex reference angle, $[\mathrm{deg}] \quad$ force constant,

\begin{tabular}{ccc} 
& & {$\left[\mathrm{mdyn} \AA / \mathrm{rad}^{2}\right]$} \\
\hline $\mathrm{C}_{\text {center }}-\mathrm{C}\left(\mathrm{sp}^{2}\right)$ & 0.000 & 1.068 \\
$\mathrm{C}\left(\mathrm{sp}^{2}\right)-\mathrm{C}_{\text {center }}$ & 0.000 & 0.147 \\
$\mathrm{C}_{\text {center }}-\mathrm{C}_{\text {center }}$ & 0.000 & 0.507 \\
\hline
\end{tabular}

\begin{tabular}{ccc}
\hline & Torsional parameter & \\
\hline torsional type & $\mathrm{n}$ & Barrier height, [kcal/mol] \\
\hline $\mathrm{C}_{\text {center }}-\mathrm{C}_{\text {center }}-\mathrm{C}_{\text {center }}-\mathrm{C}_{\text {center }}$ & $1 / 2 / 3$ & $-1.837 / 8.372 /-0.557$ \\
\hline $\mathrm{C}_{\text {center }}-\mathrm{C}_{\text {center }}-\mathrm{C}_{\text {center }}-\mathrm{C}\left(\mathrm{sp}^{2}\right)$ & $1 / 2 / 3$ & $0.640 / 4.164 /-1.720$ \\
\hline $\mathrm{C}_{\text {center }}-\mathrm{C}_{\text {center }}-\mathrm{C}\left(\mathrm{sp}^{2}\right)-\left(\mathrm{sp}^{2}\right)$ & $1 / 2 / 3$ & $-1.538 / 6.998 /-1.894$ \\
\hline $\mathrm{C}\left(\mathrm{sp}^{2}\right)-\mathrm{C}_{\text {center }}-\mathrm{C}_{\text {center }} \mathrm{C}\left(\mathrm{sp}^{2}\right)$ & $1 / 2 / 3$ & $-0.630 / 0.236 / 0.296$ \\
\hline $\mathrm{C}_{\text {center }}-\mathrm{C}\left(\mathrm{sp}^{2}\right)-\mathrm{C}\left(\mathrm{sp}^{2}\right)-\mathrm{C}\left(\mathrm{sp}^{2}\right)$ & $1 / 2 / 3$ & $1.020 / 5.473 /-1.348$ \\
\hline $\mathrm{C}_{\text {center }}-\mathrm{C}_{\text {center }}-\mathrm{C}\left(\mathrm{sp}^{2}\right)-\mathrm{H}$ & $1 / 2 / 3$ & $0.276 / 4.911 / 0.520$ \\
\hline
\end{tabular}




\section{Fragment C}

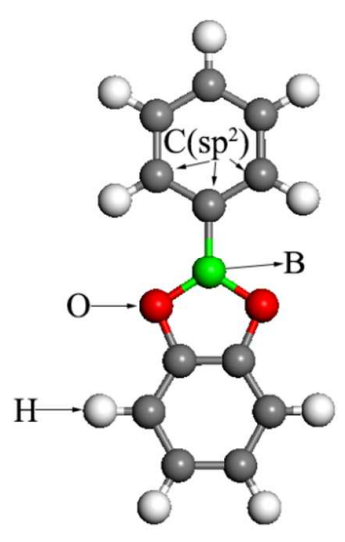

Fig.7S Fragment $\mathrm{C}$ (force field nomenclature)

Table 5S: Force field parameters for the fragment $\mathrm{C}$

Bond stretch parameter

\begin{tabular}{ccc}
\hline bond type & reference distance, $[\AA]$ & force constant, $[\mathrm{mdyn} / \AA]$ \\
\hline $\mathrm{C}\left(\mathrm{sp}^{2}\right)-\mathrm{B}$ & 1.528 & 4.79 \\
\hline $\mathrm{O}-\mathrm{B}$ & 1.389 & 4.94 \\
\hline
\end{tabular}

In-plane angle bending parameter

\begin{tabular}{ccc}
\hline Angle type & Reference angle, $[\mathrm{deg}]$ & $\begin{array}{c}\text { force constant, } \\
{\left[\mathrm{mdyn} \AA / \mathrm{rad}^{2}\right]}\end{array}$ \\
\hline $\mathrm{C}\left(\mathrm{sp}^{2}\right)-\mathrm{O}-\mathrm{B}$ & 118.200 & 0.870 \\
\hline $\mathrm{O}-\mathrm{B}-\mathrm{O}$ & 118.300 & 0.670 \\
\hline $\mathrm{C}\left(\mathrm{sp}^{2}\right)-\mathrm{B}-\mathrm{O}$ & 111.400 & 0.950 \\
\hline $\mathrm{C}\left(\mathrm{sp}^{2}\right)-\mathrm{C}\left(\mathrm{sp}^{2}\right)-\mathrm{B}$ & 125.600 & 0.330 \\
\hline $\mathrm{C}\left(\mathrm{sp}^{2}\right)-\mathrm{C}\left(\mathrm{sp}^{2}\right)-\mathrm{O}$ & 124.800 & 1.010 \\
\hline
\end{tabular}

Out-of-plane angle bending parameter

oop type: central-apex reference angle, $[\mathrm{deg}] \quad$ force constant,

\begin{tabular}{ccc} 
& {$\left[\mathrm{mdyn} \AA / \mathrm{rad}^{2}\right]$} \\
\hline $\mathrm{C}\left(\mathrm{sp}^{2}\right)-\mathrm{B}$ & 0.000 & 0.050 \\
\hline $\mathrm{B}-\mathrm{C}\left(\mathrm{sp}^{2}\right)$ & 0.000 & 0.970 \\
\hline $\mathrm{B}-\mathrm{O}$ & 0.000 & 0.990 \\
\hline $\mathrm{C}\left(\mathrm{sp}^{2}\right)-\mathrm{O}$ & 0.000 & 0.540 \\
\hline
\end{tabular}

\section{Torsional parameter}




\begin{tabular}{ccc}
\hline $\mathrm{C}\left(\mathrm{sp}^{2}\right)-\mathrm{C}\left(\mathrm{sp}^{2}\right)-\mathrm{O}-\mathrm{B}$ & 2 & 0.960 \\
$\mathrm{C}\left(\mathrm{sp}^{2}\right)-\mathrm{C}\left(\mathrm{sp}^{2}\right)-\mathrm{O}-\mathrm{B}$ & 2 & 3.890 \\
\hline $\mathrm{C}\left(\mathrm{sp}^{2}\right)-\mathrm{B}-\mathrm{O}-\mathrm{C}\left(\mathrm{sp}^{2}\right)$ & 2 & 1.120 \\
\hline $\mathrm{O}-\mathrm{B}-\mathrm{O}-\mathrm{C}\left(\mathrm{sp}^{2}\right)$ & 2 & 8.570 \\
\hline $\mathrm{C}\left(\mathrm{sp}^{2}\right)-\mathrm{C}\left(\mathrm{sp}^{2}\right)-\mathrm{C}\left(\mathrm{sp}^{2}\right)-\mathrm{O}$ & 2 & 0.000 \\
\hline $\mathrm{C}\left(\mathrm{sp}^{2}\right)-\mathrm{C}\left(\mathrm{sp}^{2}\right)-\mathrm{B}-\mathrm{O}$ & 2 & 1.500 \\
\hline
\end{tabular}

\section{Fragment D}

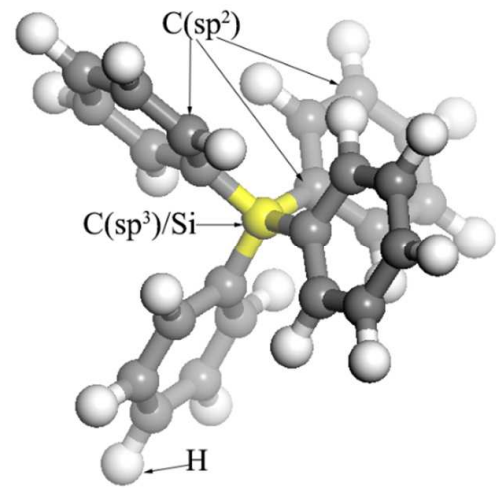

Fig.8S Tetraphenylmethane / -silane D[C/Si] (force field nomenclature)

Table 6S: Force field parameters for the Tetraphenyl-methane and -silane.

\begin{tabular}{ccc}
\hline \multicolumn{3}{c}{ Bond stretch parameter } \\
\hline bond type & reference distance, $[\AA]$ & force constant, $[\mathrm{mdyn} / \AA]$ \\
\hline $\mathrm{C}\left(\mathrm{sp}^{3}\right)-\mathrm{C}\left(\mathrm{sp}^{2}\right)$ & 1.505 & 5.096 \\
\hline $\mathrm{C}\left(\mathrm{sp}^{2}\right)-\mathrm{C}\left(\mathrm{sp}^{2}\right)$ & 1.38 & 6.56 \\
\hline $\mathrm{Si}-\mathrm{C}\left(\mathrm{sp}^{2}\right)$ & 1.262 & 9.71 \\
\hline \multicolumn{3}{c}{ In-plane angle bending parameter } \\
\hline Angle type & Reference angle, $[\mathrm{deg}]$ & force constant, \\
& & {$\left[\mathrm{mdyn} \AA / \mathrm{rad}^{2}\right]$} \\
\hline $\mathrm{C}\left(\mathrm{sp}^{2}\right)-\mathrm{C}\left(\mathrm{sp}^{3}\right)-\mathrm{C}\left(\mathrm{sp}^{2}\right)$ & 100.140 & 1.512 \\
\hline $\mathrm{C}\left(\mathrm{sp}^{2}\right)-\mathrm{Si}-\mathrm{C}\left(\mathrm{sp}^{2}\right)$ & 109.980 & 0.571
\end{tabular}

Out-of-plane angle bending parameter

oop type: central-apex reference angle, [deg] force constant,

$$
\mathrm{C}\left(\mathrm{sp}^{2}\right)-\mathrm{C}\left(\mathrm{sp}^{3}\right) \quad 0.000 \quad 0.110
$$

$\left[\mathrm{mdyn} \AA / \mathrm{rad}^{2}\right]$

Combined stretch-stretch and stretch-bend cross term parameter apex $_{1}$-central-apex 2 stretch ${ }_{1}$-bend, $[\mathrm{mdyn} \AA / \mathrm{rad}] \quad$ stretch $_{1}$ - stretch $_{2}, \mathrm{mdyn} / \AA$

$\mathrm{C}\left(\mathrm{sp}^{2}\right)-\mathrm{C}\left(\mathrm{sp}^{3}\right)-\mathrm{C}\left(\mathrm{sp}^{2}\right) \quad-0.176 \quad 0.862$

\section{Torsional parameter}




\begin{tabular}{ccc}
\hline torsional type & $\mathrm{n}$ & Barrier height, [kcal/mol] \\
\hline $\mathrm{C}\left(\mathrm{sp}^{3}\right)-\mathrm{C}\left(\mathrm{sp}^{2}\right)-\mathrm{C}\left(\mathrm{sp}^{2}\right)-\mathrm{C}\left(\mathrm{sp}^{2}\right)$ & 2 & 4.049 \\
\hline $\mathrm{Si}-\mathrm{C}\left(\mathrm{sp}^{2}\right)-\mathrm{C}\left(\mathrm{sp}^{2}\right)-\mathrm{C}\left(\mathrm{sp}^{2}\right)$ & 2 & 4.049 \\
\hline $\mathrm{C}\left(\mathrm{sp}^{2}\right)-\mathrm{C}\left(\mathrm{sp}^{2}\right)-\mathrm{C}\left(\mathrm{sp}^{2}\right)-\mathrm{C}\left(\mathrm{sp}^{2}\right)$ & 2 & 5.983 \\
\hline $\mathrm{C}\left(\mathrm{sp}^{3}\right)-\mathrm{C}\left(\mathrm{sp}^{2}\right)-\mathrm{C}\left(\mathrm{sp}^{2}\right)-\mathrm{H}$ & 2 & 5.784 \\
\hline $\mathrm{Si}-\mathrm{C}\left(\mathrm{sp}^{2}\right)-\mathrm{C}\left(\mathrm{sp}^{2}\right)-\mathrm{H}$ & 2 & 5.784 \\
\hline $\mathrm{C}\left(\mathrm{sp}^{2}\right)-\mathrm{C}\left(\mathrm{sp}^{2}\right)-\mathrm{C}\left(\mathrm{sp}^{2}\right)-\mathrm{H}$ & 2 & 6.881 \\
\hline $\mathrm{H}-\mathrm{C}\left(\mathrm{sp}^{2}\right)-\mathrm{C}\left(\mathrm{sp}^{2}\right)-\mathrm{H}$ & 2 & 5.385 \\
\hline $\mathrm{C}\left(\mathrm{sp}^{2}\right)-\mathrm{C}\left(\mathrm{sp}^{3}\right)-\mathrm{C}\left(\mathrm{sp}^{2}\right)-\mathrm{C}\left(\mathrm{sp}^{2}\right)$ & 6 & 0.000 \\
\hline $\mathrm{C}\left(\mathrm{sp}^{2}\right)-\mathrm{Si}-\mathrm{C}\left(\mathrm{sp}^{2}\right)-\mathrm{C}\left(\mathrm{sp}^{2}\right)$ & 6 & 0.0512
\end{tabular}

\section{References}

(1) Zheng, B.; Sant, M.; Demontis, P.; Suffritti, G. B. Force field for molecular dynamics computations in flexible ZIF-8 framework. J. Phys. Chem. C 2012, 116, 933-938.

(2) Zhang, X.; Jiang, J. Thermal conductivity of zeolitic imidazolate framework-8: a molecular simulation study. J. Phys. Chem. C 2013, 117, 18441-18447.

(3) Amirjalayer, S.; Snurr, R. Q.; Schmid, R. Prediction of structure and properties of boron-based covalent organic frameworks by a first-principles derived force field. J. Phys. Chem. C 2012, 116, 4921-4929. 